this without enlarging the opening. The dura mater was found to be uninjured. The bullet could not be traced, and after unsuccessful exploration with the finger and a probe the search was abandoned and the wound was stitched up. Consciousness had returned by the next day, but the patient was very drowsy and complained of severe pains in the left side of the head. The temperature was $100^{\circ} \mathrm{F}$., and there was a bloody discharge from the left ear. There was no paralysis, and the pupils were equal and reacted normally to light The temperature was normal on the third day and the patient was quite conscious, but still complaining of severe pain in the left side of the head. The discharge from the left ear was now becoming puralent. About the tifth day partial left-sided facial paralysis set in and at the same time the patient began to experience difficalty in opening his mouth. Pain in the head was persistent and there was also pain in the left temporo-maxillary articulation. The discharge from the left ear had now become purulent and the bleeding had ceased. A skiagram was taken and the bullet was found to be lying in the horizontal line of the middle ear.

I took the case over on April 10th, 1910, and found the conditions to be as follows. The man seemed dull and stupid and his speech was slow and blurred, though I attributed this to his deafness and facial paralysis rather than to any cerebral condition. There was no paralysis or weakness of the limbs, and sensation and the reflexes were normal. There was complete facial paralysis, with wasting of muscles on the left side. Deafness in the left ear was absolute, though I could not determine whether or not bone conduction was present, investigations of this kind being very difficult in uneducated natives. There was a purulent discharge from the left ear, the pus being of a leaden colour. Examined with a speculum the membrane was found to be destroyed, and deep in the tympanum a shining metallic body was seen. On passing a probe and touching this it was evident that it was the bullet. The right ear was normal in all respects. The patient was unable to open his mouth more than half an inch and complained of pain in the left temporo-maxillary articulation and in the left side of the head. The other cranial nerves were normal. The scar in the temporal region was not tender. Another skiagram was taken and the presence of the bullet was confirmed ; it was lying apparently in the middle ear.

Operation was performed on May 9th under chloroform anæsthesia. The usual incision for the radical mastoid operation was made, the flap was turned forward, and the bone was cleared. The bone was then gouged away at a rather higher level than is usually done in approaching the mastoid antrum, with the object of opening the temporal fossa. The temporal fossa was reached about half an inch below the surface. The dura mater was thickened and covered with granulation tissue, but the intra-dural pressure appeared to be normal. The cartilaginous meatus was now de'uched and a meato-conchal flap was formed. After removing some of the bone of the posterior meatal wall the tympanum came clearly into view with the bullet lying in it. The removal of bone was now proceeded with, working upwards, forwards, and inwards, until a good-sized opening into the temporosphenoidal fossa had been made. The end of the bullet could now be seen and about half of its length. After enlarging the opening a little more sufficient room was obtained to extract the bullet. It then appeared that the bullet had entered through the squamous portion of the temporal bone, passed vertically downwards between the bone and the dura mater, and finally lodged in the petrous portion of the temporal, with the posterior end in the temporo-sphenoidal fossa, the body wedged in the tympanum almost vertically and the point protruding into the glenoid fossa. The posterior wound was closed and the tympanum was plugged through the meatus.

The subsequent course of the case was uneventful. The temperature was normal next morning. The discharge from the meatus was profuse for a few days, but had lost its former leaden colour; it became less day by day and the packing of the tympanum was discontinued about a fortnight after the operation. A week later the discharge had totally ceased. The external wound healed up satisfactorily. The trismus improved daily, and three weeks after the operation the patient could open his mouth wide and could eat ordinary food. 'The headache continued for about a fortnight after the operation, becoming gradually l€ss, and then it ceased altogether.
As regards the facial paralysis and the sense of hearing, I am unable to state exactly how much improvement took place, as I went on leave about three weeks after the operation, and on my return two months later I found that the patient, as usually happens in India, had left the hospital of his own accord during my absence, and all trace of him had been lost. Three weeks after the oreration there was no improvement, but I was told that subsequently both the facial paralysis and the hearing improved decidedly. From the fact that the facial paralysis developed some days after the injury it seems almost certain that the lesion of the nerve must have been due to inflimmatory effusion and not to severance, which renders it likely that some degree of recovery would eventually take place.

The interest of this case lies in the direction and character of the wound produced by the bullet. The track of the bullet showed that, at the time the man was struck, it was falling almost vertically. Now the new short rifle is sighted up to 2750 yards, but if the rifle be elevated to an angle of $35^{\circ}$ with the horizontal, the bullet will travel 3500 to 4000 yards, and in the latter part of its trajectory will be falling almost vertically under the influence of gravity, the propulsive power of the charge being at this point practically all spent.

Lodgment in the skull generally occurs in the case of wounds inflicted by spent bullets, and the fact that this bullet entered the skull without apparently penetrating the dura mater shows that the momentum of the projectile was at the time very small. For these reasons I think that the range at which the man was hit was underestimated, and that he was probably about 3500 yards in front of the firing point, and not 2800 as I was informed. I have not access to any literature on the subject, but I think that penetration of the skull and lodgment without injury to the dura mater or brain must be almost unique, and therefore I put the case on record.

For the notes on the earlier history of the case I am indebted to Captain W. H. Riddell, I.M.S.

Jubbulpore, India.

\section{THE EYE OF SURGICAL NEEDLES: A NEW FORM.}

By J. LYNN THOMAS, C.B., F.R.C.S. ENG., SURGEON TO THE CARDIFF INFIRMARY, ETC.

I HAVE had surgical needles made for me by Messrs. Shrimpton and Fletcher of Redditch with the new eye I devised for ordinary needles some three years ago, and I have found them in practice to answer the purpose for which they were invented. The ideal requirements of an eye of a needle are not to be found in the needle, which has been in general use from the dawn of the bronze age, and which has not been materially altered for many thousands of years. Many special eyes and special needles have been made during the last few years for surgeons and for blind people, but none of them, in my opinion, possess the following necessary essentials of an ideal eye : (1) ease and simplicity in thread. ing the eye ; (2) means of fixing the sewing material in the eye to prevent it slipping when used single ; and (3) ease of unthreading. Does any existing eye meet these requirements?

I have used for many years the following needles:1. The "Kratz" or calyx-eyed needle: the frequent slipping of the thread is its annoying drawback. 2. The "Reverdin" needle I have found extremely handy for certain work, but one must work with a trained assistant in order to get the advantage in speed over that of an ordinary eye. 3. The "Doyen" eye : I have found the field for it: use too limited owing to its large size, and, also, to the rigid sides of the tapering lance-sbaped eye not allowing gripping of the silk or thread. 4. The "Paterson" eye meets all the essentials of an ideal eye, but it retains the drawbacks of the antique process of "threading the needle." Every other variety of eye which I have tried, such as the lateral sloteye or "side entrance spring-eye," possesses either a positive disadvantage over the ordinary eye or an improvement which I am quite unable to appreciats.

The eye to which I draw attention has not only a novelty in construction, which will be easily ap reciated from the 
accompanying illustrations, but the method of threading the needle, which I find to be the best (see Fig. 1), perhaps ought to be described as "needling the thread," because the part played by the thread in the process is absolutely passive, which is a reversal of the part taken by the thread in the

FIG. 1.

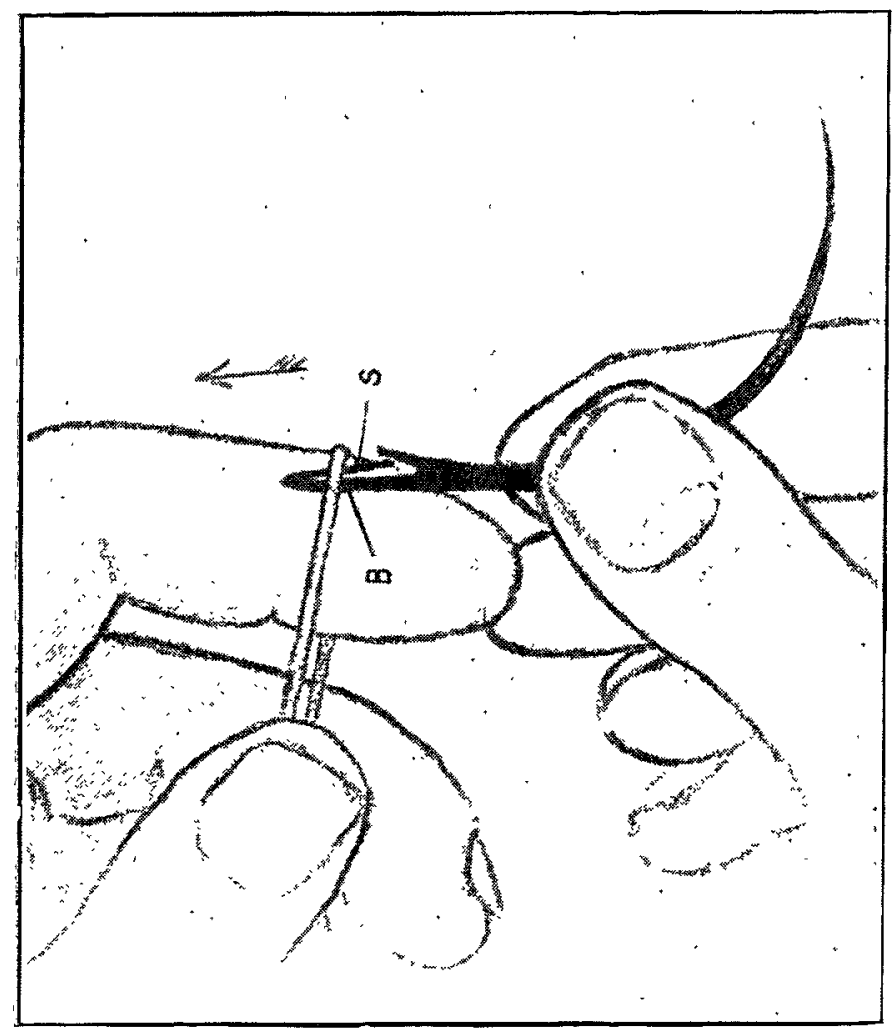

Picking up the thread.

ordinary course of "threading the needle." A further description of the eye is superfluous on account of the illustrations, except perhaps to summarise it as possessing "a side entrance" with a "spring-grip," and to state that the projection against which the spring is checked is made

Fig. 2.

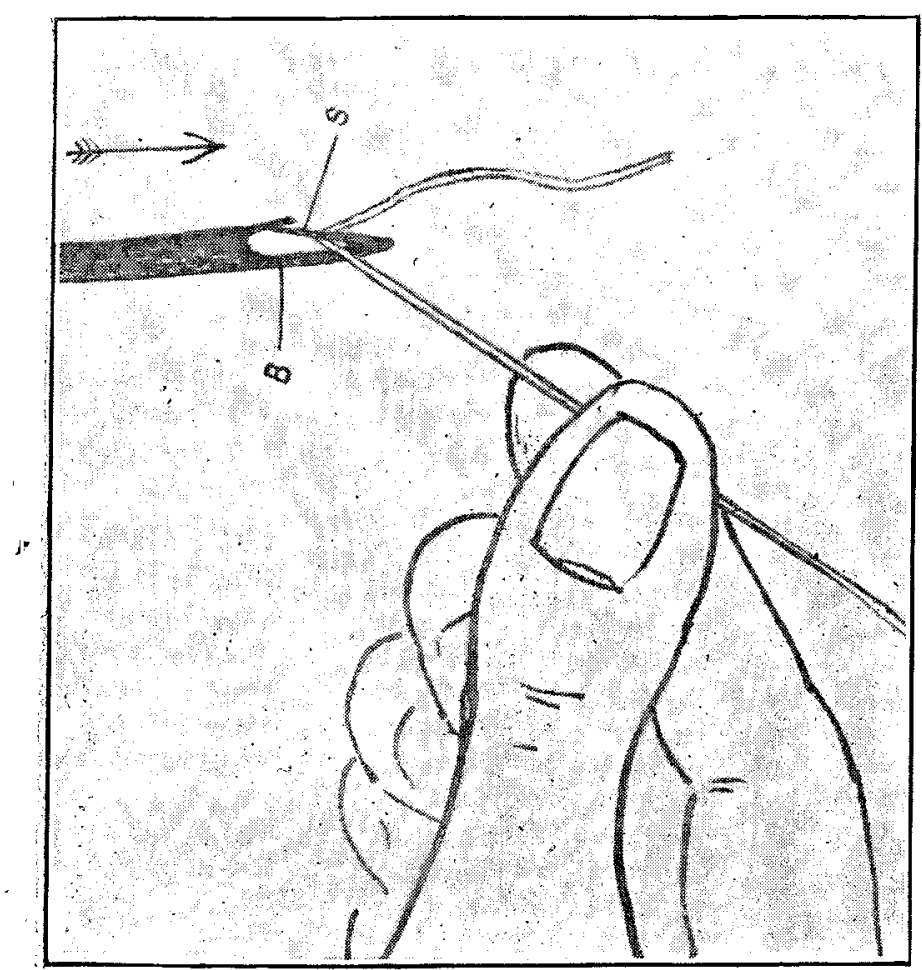

Fixing the thread.

prominently enough to be felt by emmetropes and the blind. The vanishing point between the spring $s$ and the diminutive part of the body of the needle is allows any size of thread to be firmly gripped between $\mathrm{S}$ and $\mathrm{B}$, which can enter the " side entrance."

The makers have made for me ten different sizes of needles with special forms for skin and hernia operations, and also some with Mr. Moynihan's curve, which have pleased me much both in closing deep incisions and in restoring abdominal wounds to their normal stratified condition. The arrows in the illustrations indicate the direction of the movements of the needle in "needling the thread," Fig. 1 in picking up the thread, and Fig. 2 in fixing it.

$=$ Cardiff.

\section{Clinital holtes:}

\section{MEDICAL, SURGICAL, OBSTETRICAL, AND THERAPEUTICAL.}

\section{NOTES ON A CASE OF MALIGNANT SYPHILIS.}

By W. Fingland, L.R.C.P. \& S. Edin.

ON Dec. 2nd, 1908, I was consulted by a man, aged 51 years, who three weeks previously had contracted syphilis, and exhibited a primary sore on the corona glandis, asso. ciated with well-marked inguinal adenitis. Mercurial treatment was commenced at once-pil. hydrargyri gr. i., ferri sulph. exsiccati gr. ii., in pill. Six weeks thereafter secondary symptoms made their appearance by a pustular eruption, very generally distributed, but more plentiful on the scalp, nape of neck, and face, which simulated very closely discrete variola. Recognising that the case was one of more than ordinary severity, I instituted a series of in. unctions of mild mercurial ointment with an excellent result, the eruption disappearing, excepting on some parts of the neck and scalp. The fauces and mouth were unaffected, but an acute periostitis of the anterior half. of the hard palate occurred, resulting in the necrosis of the alveoli, with the loss of two right upper incisors. This condition was treated locally with carbolic acid and camphor, equal parts. An eruption of a bullous character now appeared, with considerable destruction of tissue, sparsely distributed on the scalp, face, upper and lower limbs, which was followed by a rupial eruption which covered the shoulders, back, buttocks, and left arm. This latter eruption was very copious, the rupial crusts also being as large as a florin, many of which were necrotic and hæmorrhagic. The crusts were removed by the local application of warm carbolic oil and the sites thereof with unguentum hydrargyri subchloridi. This treatment was carried out by a trained nurse and occupied several weeks. The general health of the patient now began to be so seriously affected that specific treatment had to be suspended and measures of a restorative character resorted to-Ferri et ammoniæ citratis gr. $x$., liq. strychniæ $\dddot{m} v$., infusi calumbæ ऊss.; and later Robin's glycerophosphate syrup. $\mathrm{He}$ gradually began to improve in health, and after a few weeks of change of air and rest was able to resume work. Hydrargyri biniodidi gr. $\frac{1}{8}$ thrice daily, with potassii iodidi 3 ss. at bedtime, was prescribed, which was replaced latterly by intramuscular injections of iodipin.

On June 27th, 1909, I saw the patient at his lodgings, and was informed that he had experienced great difficulty in returning home that day. His sight and speech were affected, with paresis of both lower extremities. These symptoms becoming worse, he was removed to hospital. The patient gradually lapsed into a state of profound coma. Sight, speech, and hearing were lost ; paralysis of both lower extremities became complete, with retention of urine. He remained in this condition, dying 12 days after admission to hospital, and seven months after the appearance of the primary lesion.

Necropsy.-The following is a summary of the postmortem examination report. "Brain: Macroscopically there was a patch of recent hæmorrhagic encephalitis in the pons below the aqueduct of Sylvius. The membranes appeared to be normal. Microscopically there is thrombosis of some of the intracerebral vessels, with a large area of hæmorrhage in the brain substance, which is not of very recent origin, on account of the presence of blood pigment. The perivascular sheaths of some of the intracerebral vessels are infiltrated with small 